\title{
A Stochastic Optimal Control Theory to Model Spontaneous Breathing
}

\author{
Kyongyob Min \\ Respiratory Division, Department of Internal Medicine, Itami City Hospital, Itami, Japan \\ Email: in1007@poh.osaka-med.ac.jp
}

Received September 1, 2013; revised October 1, 2013; accepted October 8, 2013

Copyright (C) 2013 Kyongyob Min. This is an open access article distributed under the Creative Commons Attribution License, which permits unrestricted use, distribution, and reproduction in any medium, provided the original work is properly cited.

\begin{abstract}
Respiratory variables, including tidal volume and respiratory rate, display significant variability. The probability density function (PDF) of respiratory variables has been shown to contain clinical information and can predict the risk for exacerbation in asthma. However, it is uncertain why this PDF plays a major role in predicting the dynamic conditions of the respiratory system. This paper introduces a stochastic optimal control model for noisy spontaneous breathing, and obtains a Shrödinger's wave equation as the motion equation that can produce a PDF as a solution. Based on the lobules-bronchial tree model of the lung system, the tidal volume variable was expressed by a polar coordinate, by use of which the Shrödinger's wave equation of inter-breath intervals (IBIs) was obtained. Through the wave equation of IBIs, the respiratory rhythm generator was characterized by the potential function including the PDF and the parameter concerning the topographical distribution of regional pulmonary ventilations. The stochastic model in this study was assumed to have a common variance parameter in the state variables, which would originate from the variability in metabolic energy at the cell level. As a conclusion, the PDF of IBIs would become a marker of neuroplasticity in the respiratory rhythm generator through Shrödinger's wave equation for IBIs.
\end{abstract}

Keywords: Biological Variability; Stochastic Processes; Optimal Stochastic Control Theory; Probability Density Function; Shrödinger's Wave Equation

\section{Introduction}

Classical physiology is grounded on the principle of homeostasis, in which regulatory mechanisms act to reduce variability and to maintain a steady state [1]. Cherniack et al. [2] applied a systems engineering approach to the control of respiration, describing a controller (brain stem respiratory pattern generator), sensors (chemo- and mechanoreceptors), and a plant (airways, chest wall, muscles, and pulmonary tissue). With this model, fluctuations are often dismissed as "noise" of little or no significance. However, since many systems in nature, including respiration, operate away from an equilibrium point, the importance of taking fluctuations into account was well known from early models of the respiratory control mechanism. For example, measured interbreath intervals of a preterm baby at 39 and 61 weeks of postconceptional age have shown that the baby's breathing pattern was highly irregular at 39 weeks, and that the fluctuations were significantly reduced by 61 weeks [2].

For constructing realistic models of control mechanisms with biological variability in spontaneous breathing, one is faced with the problem of finding suitable ways to characterize them. A characteristic feature of fluctuations is the impossibility of precisely predicting their future values, and thus some researchers have tried to use statistical concepts to model fluctuations. From this statistical viewpoint, Frey et al. and Suki have suggested three points on noisy biological variables: 1) the fluctuations obey their own probability distribution; 2) irregular fluctuations can carry information through the probability distribution; and 3) the probability distribution may be sensitive to physiological or pathological changes $[3,4]$. Thus, to define the physiological or pathological meaning of biological variability, it is important to show why the probability distribution of noisy breathing variables is sensitive to physiological or pathological changes.

This paper introduces a stochastic optimal control theory to model spontaneous breathing. By implementing a stochastic process, the method reveals that the probability density function of noisy spontaneous breathing obeys a Shrödinger's wave function, which was introduced for 
describing motions of a quantum particle. Based on the wave function for noisy breathing, this paper concludes that the probability density function of inter-breath intervals will be a marker of neuroplasticity in the central rhythm generator.

\section{Differentiable Stochastic Processes [5]}

\subsection{Fluctuations as a Sequence of Random Variables}

A characteristic feature of fluctuations is the impossibility of precisely predicting their values. A successful attempt is to model a disturbance as a sequence of random variables or a stochastic process. A stochastic process can be defined as a family of random variables

$\left\{X(t), t=t_{0}, t_{0}+1, \cdots\right\}$. It is possible to assume that the random variables $X(t)$ represent values on the real line or in an n-dimensional Euclidean space. A stochastic process is a function of two arguments $\{X(t, \omega)\}$, where $\omega$ belongs to the sample space $\Omega$. For fixed $t, X(t, \cdot)$ is a random variable and for fixed $\omega, X(\cdot, \omega)$ is a function of time which is called a sample function or a trajectory. The trajectories can be regarded as elements of the sample function space $\Omega$. For ordinary random variables whose sample function spaces are Euclidean spaces, probability measures can be assigned by ordinary distribution functions and denoted by $P$.

Let us assign a probability function to the multidimensional random variable for any $k$ and arbitrary time $t_{j}$ with a distribution function $F$ as follows,

$$
\begin{aligned}
& F\left(\xi_{1}, \xi_{2}, \cdots, \xi_{k} ; t_{1}, t_{2}, \cdots, t_{k}\right) \\
= & P\left\{X\left(t_{1}\right) \leq \xi_{1}, X\left(t_{2}\right) \leq \xi_{2}, \cdots, X\left(t_{k}\right) \leq \xi_{k}\right\}
\end{aligned}
$$

which satisfies the conditions of symmetry in all pairs $\left(\xi_{j}, t_{j}\right)$ and consistency. The consistency condition is expressed by

$$
\begin{aligned}
& F\left(\xi_{1}, \xi_{2}, \cdots, \xi_{k} ; t_{1}, t_{2}, \cdots, t_{k}\right) \\
= & \lim _{t_{k} \rightarrow \infty} F\left(\xi_{1}, \xi_{2}, \cdots, \xi_{k} ; t_{1}, t_{2}, \cdots, t_{k}\right) .
\end{aligned}
$$

Thus, the mean value of a stochastic process $m(t)$ is defined by use of the probability distribution density $\mathrm{d} F(\xi, t)$ as follows,

$$
m(t)=\int_{-\infty}^{+\infty} \xi \mathrm{d} F(\xi, t)=E[X(t)] .
$$

The symbol $E[$ ] denotes expectation, that is, integration with respect to the measure $P$. The covariance of $X(s)$ and $X(t)$ are also given by

$$
\begin{aligned}
r(s, t) & =\operatorname{cov}[X(s), X(t)] \\
& =E[\{X(s)-m(s)\}\{X(t)-m(t)\}]
\end{aligned}
$$

When both the mean value function $m(t)$ and the covariance $r(s, t)$ exist, the stochastic process is said to be of second order.

\subsection{A Wiener Process and a Markov Process}

Let us consider the stochastic process of second order $\left\{X\left(t_{j}\right), j=1,2,3, \cdots, k\right\}$, and $t_{1}<t_{2}<t_{3}<\cdots<t_{k}$. When the set elements

$$
\begin{aligned}
& \left\{X\left(t_{k-1}\right)-X\left(t_{k}\right), X\left(t_{k-1}\right)-X\left(t_{k-2}\right), \cdots,\right. \\
& \left.X\left(t_{2}\right)-X\left(t_{1}\right), X\left(t_{1}\right)\right\}
\end{aligned}
$$

are mutually independent, the process is called a process with independent increments. If the variables are only uncorrelated, the process $\{X(t)\}$ is called a process with uncorrelated or orthogonal increments. A Wiener process is one with orthogonal increments defined by the following conditions: 1) $X(0)=0$, 2) $X(t)$ is normal, 3) $m(t)=0$ for all $t>0$, and 4) the process has independent stationary increments. Since a Wiener process has independent stationary increments and $X(0)=0$, the variance of the process is $\operatorname{var}[X(t)]=\sigma_{X}^{2}(t)=c t$, and the covariance of the process is $r(s, t)=c \times$ (the minimal difference between $t$ and $s$ ), where the parameter $c$ is called the variance parameter.

A stochastic process $\{X(t)\}$ is called a Markov process if

$$
\begin{aligned}
& P\left\{X(t) \leq \xi \mid X\left(t_{1}\right), X\left(t_{2}\right), \cdots, X\left(t_{k}\right)\right\} . \\
= & P\left\{X(t) \leq \xi \mid X\left(t_{k}\right)\right\}
\end{aligned}
$$

where $P\left\{\mid X\left(t_{k}\right)\right\}$ denotes the conditional probability given $X\left(t_{k}\right)$. When the initial probability distribution $F\left(\xi_{1}, t_{1}\right)=P\left\{X\left(t_{1}\right) \leq \xi_{1}\right\}$ and the transitional probability distribution $F\left(\xi_{t}, t \mid \xi_{s}, 3\right)=P\left\{X(t) \leq \xi_{t} \mid X(s)=\xi_{s}\right\}$ are given, the distribution function of the trajectory $\left\{X\left(t_{1}\right), X\left(t_{2}\right), \cdots, X\left(t_{k}\right)\right\}$ is given by the Bayes' rule as follows,

$$
\begin{aligned}
& F\left(\xi_{1}, \xi_{2}, \cdots, \xi_{k} ; t_{1}, t_{2}, \cdots, t_{k}\right) \\
= & F\left(\xi_{k}, t_{k} \mid \xi_{k-1}, t_{k-1}\right) \cdots F\left(\xi_{2}, t_{2} \mid \xi_{1}, t_{1}\right) F\left(\xi_{1}, t_{1}\right)
\end{aligned}
$$

(2.2.2) shows that a Markov process is defined by both the initial probability distribution and the transition probabilities.

\subsection{Stochastic State Models}

State models, i.e., systems of first order difference or differential equations, are very convenient for the analysis of systems. An extension of this concept to stochastic state models requires that the probability distribution of the state variable $x$ at future times should be uniquely determined by the actual value of the state. If $X(t+1)$ is a 
random variable which depends on the state variable $x$ at the time $t$

$$
X(t+1)-X(t)=b(x, t)+\varepsilon(x, t) .
$$

where $b(x, t)$ and $\varepsilon(x, t)$ are the conditional mean of $X(t+1)$ and a random variable given the state variable $x$ at the time $t$. When the model (2.3.1) is a Markov process, the conditional distribution of $\varepsilon(x, t)$ given $x$ is normal and the stochastic variable $\varepsilon(x, t)$ can always be normalized by its variance $\sigma^{2}$ through a Wiener process $w(t)$ with unit variance parameter,

$$
\varepsilon(x, t)=\sigma w(t) .
$$

\subsection{Stochastic Differential Equations of State Models}

Starting with the difference

$$
X(t+h)-X(t)=b(x, t) h+o\left(h^{2}\right) .
$$

where the term $o\left(h^{2}\right)$ denotes the omit terms of higher order than 2 . One can easily obtain a stochastic difference equation by adding a disturbance $\varepsilon(x, t)$,

$$
\begin{aligned}
& X(t+h)-X(t) \\
= & b(x, t) h+[\varepsilon(x, t+h)-\varepsilon(x, t)]+o\left(h^{2}\right)
\end{aligned}
$$

When the disturbance $\{\varepsilon(x, t)\}$ is a Markov process with independent increments, the conditional distribution of $[\varepsilon(x, t+h)-\varepsilon(x, t)]$ given $x$ is normal. Hence

$$
\varepsilon(x, t+h)-\varepsilon(x, t)=\sigma[w(t+h)-w(t)] .
$$

where $\{w(t)\}$ is a Wiener process with unit variance parameter. Thus, the stochastic state model is obtained for the stochastic process $\{X(t)\}$

$$
\begin{aligned}
& X(t+h)-X(t) \\
& =b(x, t) h+\sigma[w(t+h)-w(t)]+o\left(h^{2}\right)
\end{aligned}
$$

Therefore, the expectation $E[X(t+h)-X(t)]$ and the variance $\operatorname{var}[X(t+h)-X(t)]$ are obtained as (2.4.5) and (2.4.6) respectively,

$$
\begin{aligned}
E[X(t+h)-X(t)]=b(x, t) h+o\left(h^{2}\right) . & \operatorname{var}[X(t+h)-X(t)] \\
= & \sigma^{2} E[w(t+h)-w(t)]^{2}+o\left(h^{2}\right) . \\
= & h \sigma^{2}+o\left(h^{2}\right)
\end{aligned}
$$

Then, let $h$ go to zero in (2.4.4) and one obtains the following formal expression (2.4.7)

$$
X(t+\mathrm{d} t)-X(t)=\mathrm{d} X(t)=b(x, t) \mathrm{d} t+\sigma \mathrm{d} w(t)
$$

which is called a stochastic differential equation. The function of $b(x, t)$ is called a forward drift function of the state $x$ at the time $t$.

The stochastic differential (2.4.7) is defined as the limit of (2.4.4). However, another expression is possible for $\mathrm{d} X(t)$ as follows,

$$
\mathrm{d} X(t)=X(t)-X(t-h) .
$$

The difference $[w(t)-w(t-h)]=\mathrm{d} w^{*}(t)$ is not dependent on $X(t-h)$ but on $X(t)$, and the variance of $\mathrm{d} w^{*}(t)$ is $\operatorname{var}\left[\mathrm{d} w^{*}(t)\right]=-\mathrm{d} t$. Then, another stochastic differential equation is possible as follows,

$$
X(t)-X(t-\mathrm{d} t)=\mathrm{d} X(t)=b^{*}(x, t) \mathrm{d} t+\sigma \mathrm{d} w^{*}(t) .
$$

where $b^{*}(x, t)$ is a backward drift function of the stochastic process given $x$ at the time $t$.

\section{A Stochastic Control Model of Noisy Breathing}

\subsection{State Variables in Noisy Breathing}

Spontaneous breathing is described as a series of tidal volumes or changes in respiratory rhythm. A series of tidal volumes is produced from the neural activity of the respiratory center in the brain. The neural activities of the respiratory center induce changes in the length of respiratory muscles, which are transformed into changes in the pleural pressure through the architectural properties of the ribcage. The changes in the pleural pressure are transformed to the alveolar pressure through the lung parenchyma. The alveolar pressure is transformed into airway pressure by the pulmonary lobule, and goes into the environment by producing airflows through the fractal bronchial tree (Figure 1). It is important to note in Figure 1 that there are two origins of fluctuations in this process: in the respiratory rhythm generator (the neural center of respiration) and in the fractal airway modulator (the phasic asynchronous contractions of airway smooth muscles in the lobular bronchioles) [6]. Then, based on that bronchial flow $F(t)$ is composed of $N$-number of phasic lobular flow $(q)$, a tidal volume $V_{T}$ is defined as following,

$$
\begin{aligned}
V_{T} & =\int_{0}^{\tau}|F(t)| \mathrm{d} t=\int_{0}^{\tau_{I}}\left(q \sum_{j}^{N} \delta_{j}\right) \mathrm{d} t+\int_{\tau_{I}}^{\tau}\left(q \sum_{j}^{N} \delta_{j}\right) \mathrm{d} t \\
& =q N\left\{\tau_{I} \overline{\delta_{I}}+\left(\tau-\tau_{I}\right) \overline{\delta_{E}}\right\}
\end{aligned}
$$

where $\tau_{I}$ is inspiration priod, and $\delta_{j}$ is 0 or 1 for the $j$-th lobular bronchiole. $\overline{\delta_{I}}$ and $\overline{\delta_{E}}$ are the mean value of $\left\{\delta_{j}\right\}$ during inspiration and expiration, respectively. On steady state it is presumed that $\overline{\delta_{I}}=\overline{\delta_{E}}=\bar{\delta}$ and is less than 1 or $\bar{\delta}=\sin \theta$, then $V_{T}$ is expressed by the following, 


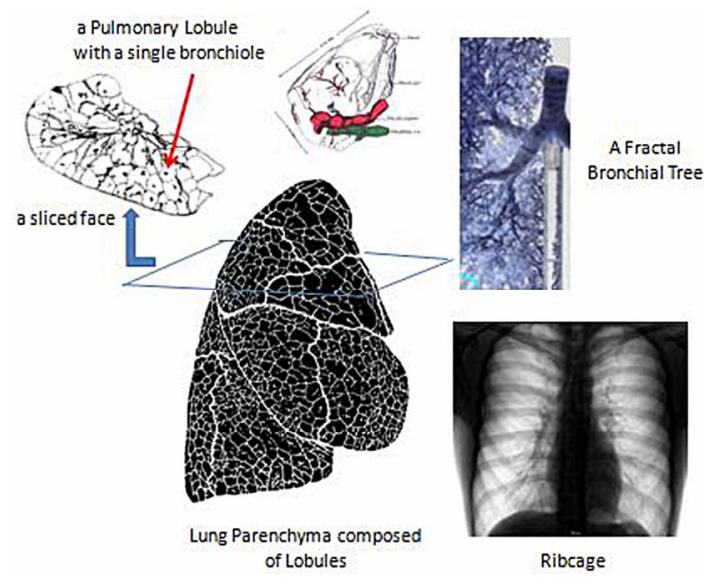

(a)

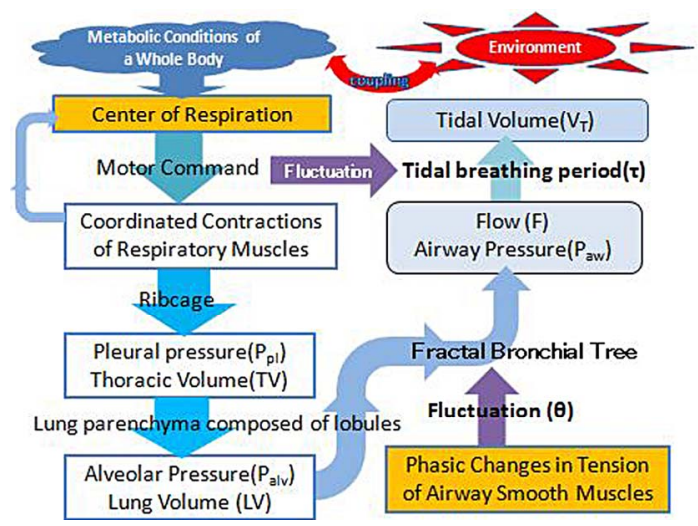

(b)

Figure 1. Components of respiratory system and producing of breathing motions. (a) Components of respiratory system: the ribcage consists of thoracic structures and the diaphragm, the right lung parenchyma consists of many lobules, a sliced face of right upper lobe lobules with a single bronchiole, and a fractal bronchial tree integrates many lobules; (b) A series of tidal volumes is produced from the neural activity of the respiratory center in the brain. The neural activities of the respiratory center induce changes in the length of respiratory muscles, which are transformed into changes in the pleural pressure through architectural properties of the ribcage. The changes in the pleural pressure are transformed into alveolar pressure through the lung parenchyma, which is composed of a large number of lobules. The alveolar pressure is transformed into airway pressure by the pulmonary lobule, and goes into the environment by producing airflows through the fractal bronchial tree, each branch of which has own bundle of smooth muscles. Bundles of airway smooth muscles dynamically change in length-tension to adapt with conditions of breathing.

$$
V_{T}=(q N \tau) \sin \theta
$$

During a voluntary forced expiration maneuver each lobule exhales a flow simultaneously. Then, the forced expiration volume in one second $\left(\mathrm{FEV}_{1.0}\right)$ is defined by the following,

$$
\mathrm{FEV}_{1.0}=\int_{0}^{1} F(t) \mathrm{d} t=q N
$$

Thus, the state variable of noisy breathing $x$ is $V_{T}$ normalized by $\mathrm{FEV}_{1.0}$ as the following,

$$
x=\frac{V_{T}}{\mathrm{FEV}_{1.0}}=\tau \sin \theta .
$$

The variable $\tau$ is the interbreath interval (IBI), and $\sin \theta$ is the proportion of simultaneously relaxed lobular bronchioles in the lung during a breath.

\subsection{A Stochastic State Model}

The spontaneous breathing is characterized by a series of respiratory variables $\left\{V_{T}\right\}$. One will consider the series $\left\{V_{T}\right\}$ as a stochastic process $\{X(t)\}$ characterized by the following stochastic equation with the state variable $x$ and the variance $\sigma^{2}$,

$$
\mathrm{d} X(t)=\left\{\begin{array}{ll}
b(x, t) \mathrm{d} t+\sigma \mathrm{d} w(t) & \mathrm{d} t>0 \\
b^{*}(x, t) \mathrm{d} t+\sigma \mathrm{d} w^{*}(t) & \mathrm{d} t<0
\end{array} .\right.
$$

where $w(t)$ and $w^{*}(t)$ are the forward Wiener process and the backward Wiener process with unit variance parameter, respectively. The function, $b(x, t)$ or $b^{*}(x, t)$ is called the forward drift function or the backward drift function of state variable $x$ at the given $t$, respectively as follows,

$$
\begin{aligned}
& b(x, t)=\lim _{\mathrm{d} t \rightarrow 0+} E_{t}\left[\frac{\mathrm{d} X(t)}{\mathrm{d} t}\right]=\lim _{\mathrm{d} t \rightarrow 0+} E_{t}\left[\frac{X(t+\mathrm{d} t)-X(t)}{\mathrm{d} t}\right] . \\
& b^{*}(x, t)=\lim _{\mathrm{d} t \rightarrow 0-} E_{t}\left[\frac{\mathrm{d} X(t)}{\mathrm{d} t}\right]=\lim _{\mathrm{d} t \rightarrow 0-} E_{t}\left[\frac{X(t)-X(t-\mathrm{d} t)}{\mathrm{d} t}\right] .
\end{aligned}
$$

where $E_{t}[]$ denotes the conditional expectation of stochastic variables at the given $t$.

\subsection{Optimal Controlled Conditions of the Stochastic State Model}

Optimal control deals with the problem of finding a control law for a given system such that a certain optimality criterion is achieved. The optimality criterion includes a value of $H$ similar to the total energy of a mechanical system. In the case of noisy breathing, a cost function $H(t)$ should be of equilibrium at optimal controlled conditions as follows,

$$
\begin{aligned}
& H(t)=E\left[\frac{1}{2}\left\{\frac{b(x, t)^{2}}{2}+\frac{b^{*}(x, t)^{2}}{2}\right\}+U(x)\right] \\
& \frac{\mathrm{d} H(t)}{\mathrm{d} t}=0
\end{aligned}
$$


where $U(x)$ is a potential function of the respiratory system. By use of the probability density function $\rho(x, t)$, the stochastic optimal controlled conditions are expressed by the following,

$$
\frac{\mathrm{d}}{\mathrm{d} t} \int\left[\frac{1}{2}\left\{\frac{b(x, t)^{2}}{2}+\frac{b^{*}(x, t)^{2}}{2}\right\}+U(x)\right] \rho(x, t) \mathrm{d} x=0 .
$$

\subsection{Einstein's Diffusion Equation}

Consider a function $f$ a continuous real valued function. The variable $f(X(t))$ is also a stochastic variable. Based on the definitions of stochastic differentials, two differentials for $f(X(t))$ are defined by use of the state variable $x$ as follows,

$$
\begin{aligned}
& \lim _{\mathrm{d} t \rightarrow 0+} E_{t}\left[\frac{\mathrm{d} f(X(t))}{\mathrm{d} t}\right] \\
= & \lim _{\mathrm{d} t \rightarrow 0+} E_{t}\left[\frac{f(X(t+\mathrm{d} t))-f(X(t))}{\mathrm{d} t}\right] \\
= & b(x, t) \frac{\mathrm{d} f(x)}{\mathrm{d} x}+\frac{\sigma^{2}}{2} \frac{\mathrm{d}^{2} f(x)}{\mathrm{d} x^{2}} \\
& \lim _{\mathrm{d} t \rightarrow 0-} E_{t}\left[\frac{\mathrm{d} f(X(t))}{\mathrm{d} t}\right] \\
= & \lim _{\mathrm{d} t \rightarrow 0-} E_{t}\left[\frac{f(X(t))-f(X(t-d t))}{\mathrm{d} t}\right] \\
= & b^{*}(x, t) \frac{\mathrm{d} f(x)}{\mathrm{d} x}-\frac{\sigma^{2}}{2} \frac{\mathrm{d}^{2} f(x)}{\mathrm{d} x^{2}}
\end{aligned}
$$

Thus, the differential of $E[f(X(t))]$ by $t$ is expressed as follows,

$$
\begin{aligned}
& \frac{\mathrm{d}}{\mathrm{d} t} E[f(X(t))] \\
= & \lim _{\mathrm{d} t \rightarrow 0+} \frac{E[f(X(t+\mathrm{d} t))]-E[f(X(t))]}{\mathrm{d} t} \\
= & \lim _{\mathrm{d} t \rightarrow 0+} E\left[E_{t}\left[\frac{f(X(t+\mathrm{d} t))-f(X(t))}{\mathrm{d} t}\right]\right] \\
= & E\left[b(x, t) \frac{\mathrm{d} f(x)}{\mathrm{d} x}+\frac{\sigma^{2}}{2} \frac{\mathrm{d}^{2} f(x)}{\mathrm{d} x^{2}}\right]
\end{aligned}
$$

When the series of stochastic variables $\{X(t)\}$ have a probability density function $\rho(x, t)$ of state variable $x$, the differential of $E[f(X(t))]$ by $t$ is also expressed as follows,

$$
\begin{aligned}
& \frac{\mathrm{d}}{\mathrm{d} t} E[f(X(t))]=\frac{\mathrm{d}}{\mathrm{d} t} \int f(x) \rho(x, t) \mathrm{d} x \\
= & \int f(x) \frac{\partial \rho(x, t)}{\partial t} \mathrm{~d} x
\end{aligned}
$$

Comparing (3.4.1) and (3.4.2), the following relation is necessary if the function $f(x)$ is arbitrary,

$$
\frac{\partial \rho(x, t)}{\partial t}=-\frac{\mathrm{d}}{\mathrm{d} x}(b(x, t) \rho(x, t))+\frac{\sigma^{2}}{2} \frac{\mathrm{d}^{2}}{\mathrm{~d} x^{2}} \rho(x, t) .
$$

Starting from (3.3.1b), the following equation is also necessary,

$$
\frac{\partial \rho(x, t)}{\partial t}=-\frac{\mathrm{d}}{\mathrm{d} x}\left(b^{*}(x, t) \rho(x, t)\right)-\frac{\sigma^{2}}{2} \frac{\mathrm{d}^{2}}{\mathrm{~d} x^{2}} \rho(x, t) .
$$

By combining (3.4.3a) and (3.4.3b), two equations are obtained as follows,

$$
\begin{gathered}
\frac{\partial \rho(x, t)}{\partial t}+\frac{\mathrm{d}}{\mathrm{d} x}\left\{\frac{1}{2}\left(b(x, t)+b^{*}(x, t)\right)\right\}=0 . \\
\frac{\mathrm{d}}{\mathrm{d} x}\left\{\frac{1}{2}\left(b(x, t)-b^{*}(x, t)\right) \rho(x, t)\right\}=\frac{\sigma^{2}}{2} \frac{\mathrm{d}^{2}}{\mathrm{~d} x^{2}} \rho(x, t) .
\end{gathered}
$$

Here, let us introduce two functions, $v(x, t)$ and $u(x, t)$ as follows

$$
\begin{aligned}
& v(x, t)=\frac{1}{2}\left\{b(x, t)+b^{*}(x, t)\right\} \\
& u(x, t)=\frac{1}{2}\left\{b(x, t)-b^{*}(x, t)\right\}
\end{aligned}
$$

Then, the functional relationships of $v(x, t), u(x, t)$ $\rho(x, t)$ can be established by the following two equations,

$$
\begin{array}{r}
\frac{\partial \rho(x, t)}{\partial t}+\frac{\mathrm{d}}{\mathrm{d} x}(v(x, t) \rho(x, t))=0 . \\
\frac{\mathrm{d}}{\mathrm{d} x}(u(x, t) \rho(x, t))=\frac{\sigma^{2}}{2} \frac{\mathrm{d}^{2}}{\mathrm{~d} x^{2}} \rho(x, t) .
\end{array}
$$

(3.4.6b) is equal to the diffusion equation of Einstein as follows

$$
u(x, t)=\frac{\sigma^{2}}{2} \frac{\mathrm{d}}{\mathrm{d} x} \log \rho(x, t) .
$$

\section{Motion Equations for Noisy Breathing}

\subsection{Shrödinger's Wave Equation as Optimal Controlled Conditions}

According to (3.3.1), the optimal condition of noisy breathing is defined using of functions $v(x, t), u(x, t)$ and $\rho(x, t)$ as follows

$$
\frac{\mathrm{d}}{\mathrm{d} t} \int\left\{\frac{1}{2}\left(v(x, t)^{2}+u(x, t)^{2}\right)+U(x)\right\} \rho(x, t) \mathrm{d} x=0 .
$$

It is possible to transform (4.1.1) to the following 
equation (see Appendix for details regarding the reference [7]),

$$
\begin{aligned}
\frac{\partial v(x, t)}{\partial t}= & \left(u(x, t) \frac{\mathrm{d}}{\mathrm{d} x} u(x, t)+\frac{\sigma^{2}}{2} \frac{\mathrm{d}^{2}}{\mathrm{~d} x^{2}} u(x, t)\right) \\
& -v(x, t) \frac{\mathrm{d}}{\mathrm{d} x} v(x, t)-\frac{\mathrm{d}}{\mathrm{d} x} U(x)
\end{aligned}
$$

According to Einstein's diffusion equation of (3.3.7), the following equation is obtained,

$$
\begin{aligned}
& \frac{\partial u(x, t)}{\partial t}=\frac{\sigma^{2}}{2} \frac{\mathrm{d}}{\mathrm{d} x} \frac{\partial \log \rho(x, t)}{\partial t} \\
= & \frac{\sigma^{2}}{2} \frac{\mathrm{d}}{\mathrm{d} x}\left\{\frac{\partial \rho(x, t)}{\partial t} / \rho(x, t)\right\} \\
= & -\frac{\sigma^{2}}{2} \frac{\mathrm{d}}{\mathrm{d} x}\left\{\frac{\frac{\mathrm{d}}{\mathrm{d} x}(v(x, t) \rho(x, t))}{\rho(x, t)}\right\} \\
= & -\frac{\sigma^{2}}{2} \frac{\mathrm{d}}{\mathrm{d} x}\left\{\frac{\mathrm{d} v(x, t)}{\mathrm{d} x}+v(x, t) \frac{\mathrm{d} \rho(x, t)}{\rho(x, t)}\right. \\
= & -\frac{\sigma^{2}}{2} \frac{\mathrm{d}^{2} v(x, t)}{\mathrm{d} x^{2}}-\frac{\mathrm{d}}{\mathrm{d} x}(v(x, t) u(x, t))
\end{aligned}
$$

The probability density function $\rho(x, t)$ obeys the Fokker-Planck equation as follows,

$$
\frac{\partial \rho(x, t)}{\partial t}=-\frac{\mathrm{d}}{\mathrm{d} x}(b(x, t) \rho(x, t))+\frac{\sigma^{2}}{2} \frac{\mathrm{d}^{2} \rho(x, t)}{\mathrm{d} x^{2}} .
$$

A set of transformations are applied to the functions $v(x, t)$ and $u(x, t)$ as follows,

$$
\begin{gathered}
v(x, t)=\sigma^{2} \frac{\mathrm{d}}{\mathrm{d} x} S(x, t) \\
u(x, t)=\frac{\sigma^{2}}{2} \frac{\mathrm{d}}{\mathrm{d} x} \log \rho(x, t)
\end{gathered}
$$

Then, from (4.1.2), (4.1.3) and (4.1.4) a set of partial differential equations are obtained as follows,

$$
\begin{gathered}
\frac{\partial S(x, t)}{\partial t} \\
=-\frac{U(x)}{\sigma^{2}}-\frac{\sigma^{2}}{2}\left[\frac{\mathrm{d}}{\mathrm{d} x} S(x, t)\right]^{2}+\frac{\sigma^{2}}{2}\left(\frac{\frac{\mathrm{d}^{2}}{\mathrm{~d} x^{2}} \sqrt{\rho(x, t)}}{\sqrt{\rho(x, t)}}\right) . \\
\text { (4.1.5a) } \\
\frac{\partial \rho(x, t)}{\partial t}=-\sigma^{2} \frac{\mathrm{d} \rho(x, t)}{\mathrm{d} x} \frac{\mathrm{d} S(x, t)}{\mathrm{d} x}-\sigma^{2} \rho(x, t) \frac{\mathrm{d}^{2} S(x, t)}{\mathrm{d} x^{2}} .
\end{gathered}
$$

Let us introduce a complex function $\psi(x, t)$ as follows,

$$
\psi(x, t)=\sqrt{\rho(x, t)} \exp (i S(x, t))
$$

By use of $\psi(x, t)$, the Equations (4.1.5a) and (4.1.5b) can be transformed to a single motion equation which equates to Shrödinger's wave equation as follows (according to reference [7]),

$$
-i \sigma^{2} \frac{\partial \psi(x, t)}{\partial t}=-\frac{\sigma^{4}}{2} \frac{\mathrm{d}^{2} \psi(x, t)}{\mathrm{d} x^{2}}+U(x) \psi(x, t) .
$$

\subsection{Distribution of Temporal and Regional Lung Ventilations}

While noisy breathing is in the steady state of optimal controlled conditions, the cost function would be equal to an optimal value of $H$. Thus, the wave function of noisy ventilations is defined by the following

$$
\left\{-\frac{\sigma^{4}}{2} \frac{\mathrm{d}^{2}}{\mathrm{~d} x^{2}}+U(x)\right\} \psi(x)=H \psi(x) .
$$

When the state variable $x$ is expressed by (3.1.3), the operator $\frac{\mathrm{d}^{2}}{\mathrm{~d} x^{2}}$ is expressed by the following

$$
\frac{\mathrm{d}^{2}}{\mathrm{~d} x^{2}}=\frac{1}{\tau^{2}} \frac{\partial}{\partial \tau}\left(\tau^{2} \frac{\partial}{\partial \tau}\right)+\frac{1}{\tau^{2} \sin \theta} \frac{\partial}{\partial \theta}\left(\sin \theta \frac{\partial}{\partial \theta}\right)
$$

If the potential function $U(x)$ is dependent on only the variable $\tau,(4.3 .1)$ can be transformed to the Equation (4.2.3) after rewriting the wave function as $\Psi(x)=T(\tau) Y(\theta)$,

$$
\begin{aligned}
& \frac{1}{T(\tau)} \frac{\partial}{\partial \tau}\left(r^{2} \frac{\partial T(\tau)}{\partial \tau}\right)-\frac{2 r^{2}}{\sigma^{4}}(U(\tau)-H) \\
= & -\frac{1}{Y(\theta)}\left\{\frac{1}{\sin \theta} \frac{\partial}{\partial \theta}\left(\sin \theta \frac{\partial Y(\theta)}{\partial \theta}\right)\right\}
\end{aligned}
$$

Each side term of (4.2.3) contain different single parameter $\tau$ or $\theta$, thus each side term of (4.2.3) should be a constant $\lambda$. An equation for $\theta$ is obtained from the right side term of (4.2.3) as follows,

$$
\frac{1}{\sin \theta} \frac{\mathrm{d}}{\mathrm{d} \theta}\left(\sin \theta \frac{\mathrm{d} Y(\theta)}{\mathrm{d} \theta}\right)+\lambda Y(\theta)=0 .
$$

When the transformation of $\cos \theta=s$ is applied to (4.2.4), the following equation is obtained,

$$
\left(1-s^{2}\right) \frac{\mathrm{d}^{2} Y(s)}{\mathrm{d} s^{2}}-2 s \frac{\mathrm{d} Y(s)}{\mathrm{d} s}+\lambda Y(s)=0 .
$$

(4.2.5) is a Legendre equation, whose solutions are obtained as Legendre orthogonal polynomials only when $\lambda=k(k+1)$ where $k=0,1,2,3, \cdots$ as follows, 


$$
Y(\theta)=Y_{k}(s)=\sum_{j=0}^{[k / 2]} \frac{(-1)^{j}(2 k-2 j) !}{2^{k} j !(k-j) !(k-2 j) !} s^{k-2 j} .
$$

That is, each solution is dependent on $k$ as follows, $Y_{0}(s)=1, Y_{1}(s)=s, Y_{2}(s)=\left(3 s^{2}-1\right) / 2$, $Y_{3}(s)=\left(5 s^{3}-3 s\right) / 2$ and so on. One can obtain the probability density function by $\rho(s)=\left|Y_{k}(s)\right|^{2}$ with $\int_{0}^{1} \rho(s) \mathrm{d} s=1$ as shown in Figure 2. It has been suggested that $\rho(s)$ would relate to patterns of temporal and regional ventilations emerging as a result of phasic contractions of smooth muscles in the lobular bronchioles. The parameter $\lambda$ would be a marker for emerging pattern of regional ventilations in the lung.

\subsection{Shrödinger's Wave Equation for Inter-Breath Intervals}

From the left side term of (4.2.3) an equation is obtained as follows,

$$
\frac{1}{\tau^{2}} \frac{\mathrm{d}}{\mathrm{d} \tau}\left(\tau^{2} \frac{\mathrm{d} T(\tau)}{\mathrm{d} \tau}\right)+\frac{2 \tau^{2}}{\sigma^{4}}(H-U(\tau)) T(\tau)-\frac{\lambda}{\tau^{2}} T(\tau)=0 .
$$

When $P(\tau)$ is introduced as $P(\tau)=\tau T(\tau)$, the following equation is obtained as another wave equation for $P(\tau)$,

\section{Probability of Regional Ventilation}

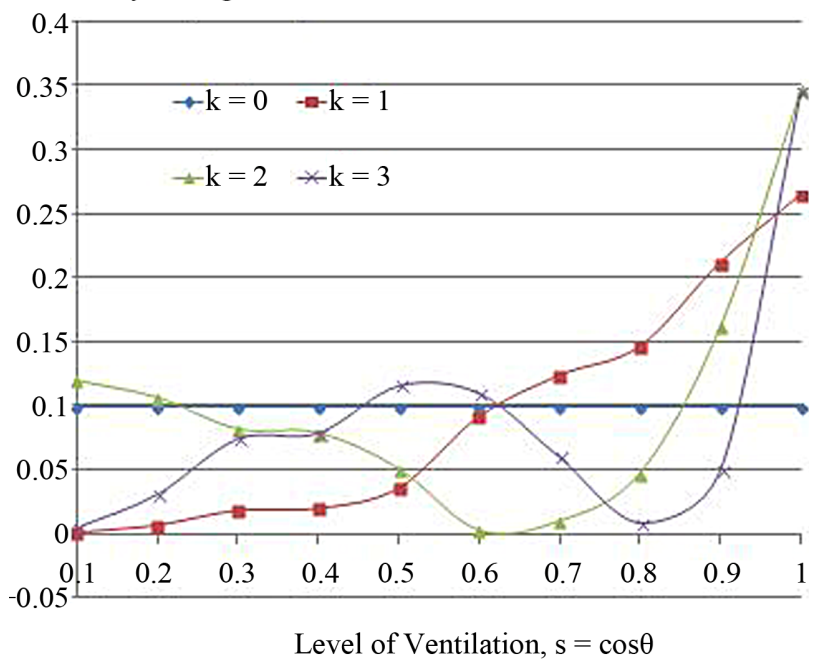

Figure 2. Probability distribution of regional ventilations in the lung. Each distribution density $\rho(s)=\left|Y_{k}(s)\right|^{2}$, where $Y_{0}(s)=1, \quad Y_{1}(s)=s, \quad Y_{2}(s)=\left(3 s^{2}-1\right) / 2$, or $Y_{3}(s)=$ $\left(5 s^{3}-3 s\right) / 2$. The probability of regional ventilations was calculated by $P(s)=\int_{s-0.1}^{s} \rho(t) d t$. Note that the distribution pattern of regional ventilations is quite different according to the parameter $k$.

$$
\left(-\sigma^{4} \frac{\mathrm{d}^{2}}{\mathrm{~d} \tau^{2}}+U(\tau)+\frac{\sigma^{4} \lambda}{2 \tau^{2}}\right) P(\tau)=H P(\tau) .
$$

One can produce a distribution density by $\rho(\tau)=|P(\tau)|^{2}$ at the optimal value of $H$, which is a probability of inter-breath intervals (IBIs) observed between $\tau$ and $\tau+\mathrm{d} \tau$.

For an optimal condition of $H$, one assumes that the wave function $P(\tau)$ is expressed by two functions $\Phi(\tau)$ and $\Psi(\tau)$ as follows,

$$
\begin{aligned}
& -\sigma^{4} \frac{\mathrm{d}^{2} \Phi(\tau)}{\mathrm{d} \tau^{2}}+\left(U(\tau)+\frac{\sigma^{4} \lambda}{2 \tau^{2}}\right) \Phi(\tau)=H \Phi(\tau) . \\
& -\sigma^{4} \frac{\mathrm{d}^{2} \Psi(\tau)}{\mathrm{d} r^{2}}+\left(U(\tau)+\frac{\sigma^{4} \lambda}{2 \tau^{2}}\right) \Psi(\tau)=H \Psi(\tau) .
\end{aligned}
$$

By $(4.3 .2 \mathrm{a}) \times \Psi(\tau)-(4.3 .2 \mathrm{~b}) \times \Phi(\tau)$ calculating, one obtains the following equation:

$$
\frac{\mathrm{d}}{\mathrm{d} \tau}\left[\Phi(\tau) \frac{\mathrm{d} \Psi(\tau)}{\mathrm{d} \tau}-\Psi(\tau) \frac{\mathrm{d} \Phi(\tau)}{\mathrm{d} \tau}\right]=0 .
$$

If $\tau \rightarrow \infty$, then both $\Psi(\tau)$ and $\Phi(\tau) \rightarrow 0$. Therefore, (4.3.3) is transformed to (4.3.4) as follows,

$$
\Phi(\tau) \frac{\mathrm{d} \Psi(\tau)}{\mathrm{d} \tau}=\Psi(\tau) \frac{\mathrm{d} \Phi(\tau)}{\mathrm{d} \tau}
$$

or

$$
\Psi(\tau) \propto \Phi(\tau) .
$$

Thus, the state of the rhythm generator is uniquely determined with dependence on the value of $H$.

The probability density function $\rho$ (IBI) is expressed by the wave function in (4.3.1) as follows:

$\rho(\mathrm{IBI})=|P(\tau)|^{2}$. When the wave function $P(\tau)$ is expressed by the following,

$$
P(\tau)=\exp (-f(\tau))
$$

$U(\tau)$ is expressed by the following,

$$
U(\tau)=H-\sigma^{4}\left[\frac{\mathrm{d}^{2} f(\tau)}{\mathrm{d} \tau^{2}}-\left(\frac{\mathrm{d} f(\tau)}{\mathrm{d} \tau}\right)^{2}+\frac{\lambda}{2 \tau^{2}}\right] .
$$

The Equation (4.3.6) explains how the probability density function (PDF) relates to the function of the central rhythm generator.

\section{Discussion}

\subsection{What Is the Origin of Biological Variability?}

Biological processes in the body provide an endless and astounding source of complexity. This variability is not simply attributable to random noise superimposed on regular processes. Instead, some researchers have suggested 
that hidden in the noise are temporal structures which may be important markers of numerous acute and chronic diseases [1]. Frey et al. and Suki have suggested that the probability distribution of noisy physiological variables would have biological information [3,4]. However, little work has been done in regard to the origin of biological variability. In this study, fluctuations in respiratory state variables are assumed to be represented by a single value, $\sigma^{2}$. Based on recent in vitro experimental studies, Suki and colleagues have proposed that energetic and metabolic fluctuations at the level of the cell are essential components of biological variability [8]. If any biological variability originates from the variability of energy at the level of the cell, it will be acceptable to hypothesize that the fluctuations of physiological state variables are described by the single quantity of $\sigma^{2}$. That is, a living cell would produce biological variability through molecular fluctuations, and this biological variability could be modeled by the universal constant $\sigma^{2}$, much like the Planck constant in the quantum physics.

\subsection{Temporal and Regional Distribution of Lobular Ventilations}

By using the lobule and fractal bronchial tree model (LBT model) [6], the state variable of spontaneous noisy breathing was expressed by the polar coordinate $x$, which is composed of two dimensional variables $\tau$ and $\theta$. The biological variability of $\theta$ may be determined through the variability in the amplitude of tidal volume $V_{T}$ based on (3.1.3). The Legendre Equation (4.2.5) would describe the temporal and regional distribution of pulmonary ventilations, which Venegas et al. recently demonstrated as the images of positron emission tomography (PET) [9].

If biological variability originates at the level of the cell, the biological variability of perfusion in the lung is also expected to have the same motion equation as (4.2.5). In the case of pulmonary perfusion, it is necessary to define the state variable from the stroke volume (SV). If the biological variability in both $V_{T}$ and $\mathrm{SV}$ is measured simultaneously, one would be able to describe the ventilation-perfusion matching in the lung according to (4.2.6).

\subsection{Neuroplasticity of the Respiratory Rhythm Generator (RRG)}

Respiratory rhythm generation arises in the medullary neurons that initiate rhythmic inspiratory and expiratory activity. Several studies suggest that the pre-Bötzinger complex, a discrete group of propriobulbar neurons in the ventrolateral medulla, plays a critical role in respiration rhythm generation, although this hypothesis is not without controversy [10]. Pattern-forming neurons include premotoneurons and motoneurons in the brain stem or spinal cord, where complex activation patterns arise from interactions between their intrinsic properties and synaptic inputs. Pattern formation establishes the detailed spatio-temporal motor output of respiratory muscles, coordinating their activation to produce a breath with the appropriate characteristics. These coordinated, complex interactions among groups of neurons in the brain produce an optimal breathing rhythm which is described by $P(\tau)$ in (4.3.6).

Mitchell and Johnson have stated that a comprehensive conceptual framework of neuroplasticity in the respiratory control system is lacking [10]. However, the Equation (4.3.6) can provide a comprehensive framework for respiratory rhythm generation since this expression includes an optimal total energy $H$ of the respiratory system, the topographical distribution parameter $\lambda$ of regional ventilation in the lung, and the probability density function (PDF) of inter-breath intervals (IBIs).

Frey et al. [3] and Fadel et al. [11] demonstrated the fractal properties of PDFs of IBIs in preterm, term babies and a third of adults at rest. When there are fractal properties in PDFs of IBIs as follows $\rho(\tau) \propto \tau^{-\alpha}$, according to (4.3.6) the potential $U(\tau)$ of the RRG is expressed by the following,

$$
U(\tau)=H-\frac{\sigma^{4}}{2 \tau^{2}}[-2 \alpha(\alpha+1)+\lambda] .
$$

This potential of the RRG shows that development of the RRG in infants leads to a change in parameters $\alpha$ and $\lambda$, but no change in the structure of the potential function. If a change in the structure of the potential function signals neuroplasticity, the developmental change of the $R R G$ is not a neuroplastic process.

\section{Conclusion}

Variability in spontaneous breathing is not simply attributable to random noise superimposed on a regular respiratory process. Biological variability should originate from energetic fluctuations at the level of the cell, and thus it is acceptable to assume that biological variability is a universal constant amongst all physiological variables. Under this assumption, a stochastic state model for spontaneous noisy breathing produced Shrödinger's wave equation as the motion equation. Based on the lobule and fractal bronchial tree model of the lung, two wave equations were obtained from the Shrödinger's equation: one for the respiratory rhythm generator and another for the modulator of airway smooth muscles in the lung. From these equations, the function of the respiratory rhythm generator was defined as a complex function including probability density functions of biological variability in both rhythm and amplitude of spontaneous noisy breathing. The stochastic control model analysis in this study can thus provide a new tool applicable for the analysis of any noisy biological processes. 


\section{REFERENCES}

[1] A. L. Goldberger, "Heartbeats, Hormones, and Health: Is Variability the Spice of Life?" American Journal of Respiratory and Critical Care Medicine, Vol. 163, No. 6, 2001, pp. 1289-1290.

http://dx.doi.org/10.1164/ajrccm.163.6.ed1801a

[2] N. S. Cherniack, G. S. Longobardo, O. R. Levine, R. Mellins and A. P. Fishman, "Periodic Breathing in Dogs," Journal of Applied Physiology, Vol. 21, No. 6, 1966, pp. 1847-1854.

[3] U. Frey, M. Silverman, A. L. Barabási and B. Suki, "Irregularities and Power Law Distributions in the Breathing Pattern in Preterm and Term Infants," Journal of Applied Physiology, Vol. 85, No. 3, 1998, pp. 789-797.

[4] B. Suki, "Fluctuations and Power Laws in Pulmonary Physiology," American Journal of Respiratory and Critical Care Medicine, Vol. 166, No. 2, 2002, pp. 133-137. http://dx.doi.org/10.1164/rccm.200202-152PP

[5] K. J. Astroem, "Introduction to Stochastic Control Theory," Dover Books on Electrical Engineering, New York, 1970.

[6] K. Min, K. Hosoi, Y. Kinoshita, S. Hara, H. Degami, T. Takada and T. Nakamura, "Use of Fractal Geometry to
Propose a New Mechanism of Airway-Parenchymal Interdependence," Open Journal of Molecular and Integrated Physiology, Vol. 2, No. 1, 2012, pp. 14-20. http://dx.doi.org/10.4236/ojmip.2012.21003

[7] K. Yasue, "Quantum Mechanics and Optimal Stochastic Control Theory," Kaimei-shya, Tokyo, 2007 (in Japanese).

[8] B. Suki, N. Martinez, H. Parameswaran, A. Majumdar, R. Dellaca, C. Berry, J. J. Pillow and E. Bartolak-Suki, "Variability in the Respiratory System: Possible Origins And Implications," B29, The Lung on the Border between Order and Chaos, 2012, p. A2682.

[9] J. G. Venegas, T. Winkler, G. Musch, M. F. Vidal Melo, D. Layfield, N. Tgavalekos, A. J. Fischman, R. J. Callahan, G. Bellani and R. S. Harris, "Self-Organized Patchiness in Asthma as a Prelude to Catastrophic Shifts," Nature, Vol. 434, No. 7034, 2005, pp. 777-782. http://dx.doi.org/10.1038/nature03490

[10] G. S. Mitchell and S. M. Johnson, "Invited Review: Neuroplasticity in Respiratory Motor Control," Journal of Applied Physiology, Vol. 94, No. 1, 2003, pp. 358-374.

[11] P. J. Fadel, S. M. Barman, S. W. Phillips and G. L. Gebber, "Fractal Fluctuations in Human Respiration," Journal of Applied Physiology, Vol. 97, No. 6, 2004, pp. 20562064. http://dx.doi.org/10.1152/japplphysiol.00657.2004 


\section{Appendix}

$$
\frac{\mathrm{d}}{\mathrm{d} t} \int\left\{\frac{1}{2}\left(v(x, t)^{2}+u(x, t)^{2}\right)+U(x)\right\} \rho(x, t) \mathrm{d} x=0 .
$$

The first term of (4.1.1) is calculated as follows,

$$
\begin{aligned}
& \frac{\mathrm{d}}{\mathrm{d} t} \int \frac{1}{2} v(x, t)^{2} \rho(x, t) \mathrm{d} x=\int \frac{1}{2} \frac{\partial v(x, t)^{2} \rho(x, t)}{\partial t} \mathrm{~d} x=\int\left\{v(x, t) \frac{\partial v(x, t)}{\partial t} \rho(x, t)+\frac{1}{2} v(x, t)^{2} \frac{\partial \rho(x, t)}{\partial t}\right\} \mathrm{d} x \\
& =\int\left\{v(x, t) \frac{\partial v(x, t)}{\partial t} \rho(x, t)-\frac{1}{2} v(x, t)^{2} \frac{\mathrm{d}^{2}}{\mathrm{~d} x^{2}}(v(x, t) \rho(x, t))\right\} \mathrm{d} x=\int\left\{\frac{\partial v(x, t)}{\partial t}+v(x, t) \frac{\partial v(x, t)}{\partial t}\right\} v(x, t) \rho(x, t) \mathrm{d} x
\end{aligned}
$$

The second term of (4.1.1) is also transformed as follows,

$$
\begin{aligned}
& \frac{\mathrm{d}}{\mathrm{d} t} \int \frac{1}{2} u(x, t)^{2} \rho(x, t) \mathrm{d} x=\int \frac{1}{2} \frac{\partial u(x, t)^{2} \rho(x, t)}{\partial t} \mathrm{~d} x=\int\left\{u(x, t) \frac{\partial u(x, t)}{\partial t} \rho(x, t)+\frac{1}{2} u(x, t)^{2} \frac{\partial \rho(x, t)}{\partial t}\right\} \mathrm{d} x \\
& =\int\left\{\frac{\sigma^{2}}{2} u(x, t) \frac{\partial}{\partial t}\left(\frac{\mathrm{d}}{\mathrm{d} x} \log \rho(x, t)\right) \rho(x, t)-\frac{1}{2} u(x, t)^{2} \frac{\mathrm{d}}{\mathrm{d} x}(v(x, t) \rho(x, t))\right\} \mathrm{d} x \\
& =\int\left\{\frac{\sigma^{2}}{2} u(x, t) \frac{\frac{\mathrm{d} \rho(x, t)}{\rho(x)}}{\rho(x, t)^{2}} \frac{\mathrm{d}}{\mathrm{d} x}(v(x, t) \rho(x, t)) \rho(x, t)-\frac{\sigma^{2}}{2} u(x, t) \frac{\mathrm{d}}{\mathrm{d} x}\left(\frac{\mathrm{d} v(x, t)}{\mathrm{d} x} \rho(x, t)\right)-\frac{1}{2} u(x, t)^{2} \frac{\mathrm{d}}{\mathrm{d} x}(v(x, t) \rho(x, t))\right\} \mathrm{d} x \\
& =\int\left\{\frac{1}{2} u(x, t)^{2} \frac{\mathrm{d}}{\mathrm{d} x}(v(x, t) \rho(x, t))-\frac{\sigma^{2}}{2} u(x, t) \frac{\mathrm{d}^{2}}{\mathrm{~d} x^{2}}(v(x, t) \rho(x, t))\right\} \mathrm{d} x \\
& =-\int\left\{u(x, t) \frac{\mathrm{d}}{\mathrm{d} x} u(x, t)+\frac{\sigma^{2}}{2} \frac{\mathrm{d}^{2} u(x, t)}{\mathrm{d} x^{2}}\right\}(v(x, t) \rho(x, t)) \mathrm{d} x
\end{aligned}
$$

The third term of (4.1.1) is expressed by following,

$$
\frac{\mathrm{d}}{\mathrm{d} t} \int U(x) \rho(x, t) \mathrm{d} x=\int U(x) \frac{\partial \rho(x, t)}{\partial t} \mathrm{~d} x=-\int U(x) \frac{\mathrm{d}}{\mathrm{d} x}(v(x, t) \rho(x, t)) \mathrm{d} x=\int \frac{\mathrm{d} U(x)}{\mathrm{d} x}(v(x, t) \rho(x, t)) \mathrm{d} x
$$

By combining (A.1), (A.2) and (A.3), the criterion of optimal control is expressed by the following,

$$
\begin{aligned}
& \frac{\mathrm{d}}{\mathrm{d} t}\left\{\left\{\frac{1}{2}\left(v(x, t)^{2}+u(x, t)^{2}\right)+U(x)\right\} \rho(x, t) \mathrm{d} x\right. \\
= & \int\left[\frac{\partial v(x, t)}{\partial t}-\left(u(x, t) \frac{\mathrm{d} u(x, t)}{\mathrm{d} x}+\frac{\sigma^{2}}{2} \frac{\mathrm{d}^{2} u(x, t)}{\mathrm{d} x^{2}}\right)+v(x, t) \frac{\mathrm{d} v(x, t)}{\mathrm{d} x}+\frac{\mathrm{d} U(x)}{\mathrm{d} x}\right] v(x, t) \rho(x, t) \mathrm{d} x=0
\end{aligned}
$$

The Equation (4.1.2) is obtained as the necessity for the criterion of control (A.4) as follows,

$$
\left\{\frac{\partial v(x, t)}{\partial t}-\left(u(x, t) \frac{\mathrm{d} u(x, t)}{\mathrm{d} x}+\frac{\sigma^{2}}{2} \frac{\mathrm{d}^{2} u(x, t)}{\mathrm{d} x^{2}}\right)+v(x, t) \frac{\mathrm{d} v(x, t)}{\mathrm{d} x}\right\}+\frac{\mathrm{d} U(x)}{\mathrm{d} x}=0
$$

